\title{
miRNA-based "Fitness Score" to Assess the Individual Response to Diet, Metabolism and Exercise
}

\author{
Ulrike Dagmar Barbara Krammer \\ University of Vienna: Universitat Wien
}

\section{Sylvia Tschida}

University of Vienna Faculty of Life Sciences: Universitat Wien Fakultat fur Lebenswissenschaften Julia Berner

University of Vienna Faculty of Life Sciences: Universitat Wien Fakultat fur Lebenswissenschaften

Stephanie Lilja

University of Vienna Faculty of Life Sciences: Universitat Wien Fakultat fur Lebenswissenschaften

Olivier Jerome Switzeny

HealthBioCare Gmbh

\section{Berit Hippe}

HealthBioCare Gmbh

\section{Petra Rust}

University of Vienna Faculty of Life Sciences: Universitat Wien Fakultat fur Lebenswissenschaften Alexander Günter Haslberger ( $\boldsymbol{D}$ alexander.haslberger@univie.ac.at)

University of Vienna https://orcid.org/0000-0001-9699-537X

\section{Research article}

Keywords: exercise, epigenetic, miRNAs, biomarker, fitness score, nutrition

Posted Date: September 13th, 2021

DOI: https://doi.org/10.21203/rs.3.rs-796683/v1

License: (1) (1) This work is licensed under a Creative Commons Attribution 4.0 International License. Read Full License 


\section{Abstract}

Background: Regular, especially sustained exercise training plays an important role in the prevention and treatment of multiple chronic diseases. Some of the underlying molecular and cellular mechanisms behind the adaptive response to physical activity are still unclear, but recent findings suggest a possible role of epigenetic mechanisms, especially miRNAs, in the progression and management of exercise related changes. Due to the combination of the analysis of epigenetic biomarkers (miRNAs), the intake of food and supplements, and genetic dispositions, a "fitness sore" was evaluated to assess the individual response to nutrition, metabolism and exercise.

Methods: In response to a 12-week sport intervention we analyzed genetic and epigenetic biomarkers in capillary blood, including Line-1 methylation, three SNPs and ten miRNAs using HRM and qPCR analysis. These biomarkers were also analyzed in a control group without intervention. Food frequency intake, including dietary supplement intake, and general health questionnaires were surveyed under the supervision of trained staff.

Results: Exercise training decreased the expression of miR-20a, -22 and -505 $(p<0.02)$ and improved the "fitness score", which estimates eight different lifestyle factors to assess, nutrition, inflammation, cardiovascular fitness, injury risk, regeneration, muscle- and hydration status as well as stress level. In addition, we were able to determine correlations between individual miRNAs, miR-20a, -22 and -101 ( $\mathrm{p}<$ 0.04 ), and the genetic predisposition for endurance and / or strength and for obesity risk (ACE, ACTN3 and $F T O)$, as well as between miRNAs and the body composition $(\mathrm{p}<0.05)$. And we identified two miRNAs, miR-19b and $-378 a(p<0.05)$, which could potentially provide information about the micronutrient / vitamin requirements of an athlete.

Conclusions: Due to the detailed knowledge of individual regulatory mechanisms in the metabolism of sport intervention and / or nutritional behavior, this knowledge and our results can be used for personalized interventions and in the context of the new field of precision nutrition and precision training.

\section{Introduction}

Regular, especially sustained exercise training plays an important role in the prevention and treatment of multiple chronic diseases, such as cardiovascular diseases, metabolic disorders, neurological / cognitive diseases, musculoskeletal disorders and cancer, but also in the maintaining of health and well-being [1]. In addition, aging conditions, such as a decline in cognitive / motor functions, loss of muscle mass and strength, can be improved through regular exercise and age-related deterioration can be delayed $[2,3]$. Moreover, our genetic architecture and epigenetic manifestations play an essential role in this big area, e.g. adherence to exercise, a person's response to exercise, and metabolic adaptions through exercise training [4].

Variations in voluntary physical activity are an important determinant of long-term human health. The predisposition to voluntary activity is heritable and leads to protective metabolic changes [5]. From 
animal and human research, however, it is known that neural signaling and pleasure / reward systems in the brain to a large extent promote the tendency to be physically active and to adhere to a training program. Based on a large epidemiologic study (TIGER study), 26 single nucleotide polymorphisms (SNPs) in six candidate genes (BDNF, BDNFOS, DRD2, DRD4, HTR2A and HTR2C) were identified that are related to behavior during physical activity, i.e. to adherence, duration, intensity and total dose of exercise in young men and women [6]. On the other hand, it is assumed that there is also a genetic predisposition to the type of sport (endurance ACTN3 or strength ACE). One example is the Alpha-actinin-3 (ACTN3) genotype, which affects human performance in various ways through the absence or presence of aactinin-3 in skeletal muscle [7]. In addition, variants of the Fat mass and obesity associated (FTO) gene show a strong association with obesity and fat mass, but also described to influence the skeletal muscle phenotypes of athletes [8]. A study by Almén et al., 2012 [9] suggests that the effect of the FTO obesity risk allele may be mediated through epigenetic changes.

However, genetic dispositions are insufficient to explain the individual response to diet, nutrition and exercise. It has already been shown that exercise can alter gene expression [10] and that an early response to physical activity triggers increased transcription of regulatory, metabolic, and myogenic genes, which are important for mediating subsequent adaptations in skeletal muscle and contribute to improved fitness [4]. Through increased transcription, exercise can change the methylation status of certain genes that are involved in muscle function. And this can lead to the formation of a long-lasting favorable expression pattern for improved training ability. In response to physical activity, the methylation profile changes in a dose-dependent, gene-specific and tissue-specific manner [11, 12]. In addition, miRNAs are also changed by physical activity [13] and they can influence gene expression posttranscriptional. miRNAs bind to their miRNA response elements (MREs) situated on RNAs and upon binding initiate the degradation of the RNA and inhibit their translation, i.e. miRNAs silence specific genes, so the expression of miRNAs depends on the exercise type, duration, intensity and tissue. Therefore, miRNAs are subdivided into cardiac-, muscle-depending and circulated miRNAs (ci-miRNAs) [14, 15].

In addition to understand the various pathways that physical activity triggers in the human body, there is growing interest and the use of so-called biomarkers, which individually reflect and evaluate the health, performance and recovery of every athlete or lay persons. These informations could help athletes and / or coaches improve performance while reducing the risk of overtraining and injury. At the moment mainly proteins or electrolytes as well as genetic tests are used as biomarkers, which are measured in blood, urine or saliva [16]. By the time, these markers are detectable in the circulation, however, consequential damage such as inflammation and injuries may already have occurred. It is assumed that changes in miRNA expressions in body fluids will occur earlier than with conventional biomarkers, which is why miRNAs are more favorable for the early detection of possible deficits [17].

The main goal of our work was to identify miRNAs that are influenced by endurance and strength exercise and are suitable as biomarkers to provide information about the current fitness and health status of a person. This can even be achieved through a non-invasive method [13], which makes it easier for athletes and trainers to take samples and to counteract possible deficits immediately. Furthermore, 
another aim was to identify possible interactions between selected epigenetic and genetic markers, the diet and the anthropometric data in order to be able to make personal recommendations for athletes in the future. Since the literature almost exclusively contains sports intervention studies with men, with this study we also wanted to create a certain basis for the interactions and effects of exercise and epigenetics in women.

\section{Materials And Methods}

Marker selection: In a pilot study from 2018, we analyzed over 340 different miRNAs and two methylation sites from capillary blood from 14 healthy women, aged between 36 and 59 years, with an average BMI of $32 \mathrm{~kg} / \mathrm{m}^{2}$. The results of this study as well as literature data were used to set up and select the markers for the following sports intervention study (Figure 1).

Study design of the sports intervention study: Over 90 participants, women and men, aged between 19 and 55 years, were recruited for the sports intervention at the University of Vienna, Department of Nutritional Sciences, who were physically inactive and had a BMI between 20 and $35 \mathrm{~kg} / \mathrm{m}^{2}$ (Table S1, for further details, e.g. body composition), but were generally healthy. All study participants have given their written consent to the use of their data. Furthermore, elite athletes, individuals who participated vigorously in competitive sports (for the past 2 years) and anyone with a history of chronic illness or medication use has been excluded from participation. The participants received a 12-week training intervention (Table S4) that included both endurance and strength training. During their training sessions, they were supervised by the gym trainers and our staff and had to keep a training log. The participants were contacted weekly by phone and asked about their progress or motivated if necessary. Before (T0) and after (24 to 48 hours after the last training session, T1) the intervention, capillary blood samples (DBS) were collected, the body composition was measured (using BIA) and a questionnaire regarding diet and lifestyle was queried. 61 participants (20 men and 41 women) successfully completed the intervention and in addition, capillary blood samples were collected from 9 participants 10 month after the intervention (T2, Table S2) to see whether the expressions returned to baseline (Figure 1B).

To exclude seasonal or diet-related changes in expression patterns, 20 participants, women and men, aged between 23 and 65 years and a BMI between 20 and $30 \mathrm{~kg} / \mathrm{m}^{2}$, were examined without sports intervention or lifestyle change (Figure 1B and Table S3)

Sample collection, RNA- and DNA-extraction: The capillary blood was collected on Whatman ${ }^{\circledR}$ protein saver cards (Sigma-Aldrich, Austria) using the safety Lancet Extra 18G (Sarstedt, Germany) from the fingertip of the non-dominant hand and dried overnight. Two points with a diameter of $10 \mathrm{~mm}$ was punched out of each DBS. Then the RNA was extracted using the miRNeasy Micro Kit (Qiagen, Germany) and the DNA using the QIAamp ${ }^{\circledR}$ DNA Mini Kit (Qiagen, Germany). Samples were stored at -20 degrees. 12 RNA samples from the pilot study were sent to Qiagen in Germany for further analysis (miRCURY LNA miRNA PCR Human Panel I). 
SNP genotyping: We used the TaqMan ${ }^{T M}$ Genotyping Assays from Thermofisher, Netherlands for the analysis of the SNPs (ACTN3 (rs1815739), ACE (rs4341) and FTO (rs1121980)) under the default settings on QuantStudio ${ }^{T M}$ 3. The SNPs were then evaluated using the TaqMan Genotyper Software v1.6.

Bisulfite conversion and high-resolution melting (HRM) analysis: The DNA Samples were prepared with the EpiTect Bisulfite Kit (Qiagen, Germany) for the qPCR and HRM analysis using Rotor Gene® Q. For the Line-1 methylation analysis, we used the primers from Marques-Rocha et al., 2016 [18]. The methylation percentage was then calculated using the AUC method.

CDNA-Synthesis and real-time PCR (qPCR): We used the TaqMan ${ }^{T M}$ Advanced microRNA cDNA Synthesis Kit and TaqMan ${ }^{T M}$ Advanced microRNA assays under the default settings on QuantStudio ${ }^{T M} 3$ from Thermofisher, Netherlands. The cDNA samples were stored at -20 degrees. In the pilot study, miR-21 and miR-155 were analyzed in addition to the Qiagen panel. Based on this study, eight miRNAs (miR-19b, -20a, $-22,-30 e,-101,-146 a,-378 a$ and -505$)$ were then included and analyzed in the sports intervention study 2019 - 2020. Furthermore, two reference genes (miR-24 and -93) were examined for the evaluation of the expression patterns (using $\Delta \Delta$ Ct method) of the eight selected miRNAs. The reference genes were selected based on the pilot study and through the literature.

Statistical analysis: The statistical evaluation was carried out using IBM SPSS statistics 20 and GraphPad Prism 6. The relevant tests were students t-test, Pearson's and Spearman's Rho correlation, Mann-Whitney U-test, Kendall's Tau and one-factorial ANOVA including Post-Hoc test (Bonferroni and Scheffé) and linear regression. For all tests, a p-value equal or less than 0.05 was assumed to be significant.

\section{Results}

\subsection{Pilot study 2018}

Eight of the miRNAs (miR-494, -369, -122, -146a, -30e, -135a, -22 and -20a) measured in the miRCURY LNA miRNA PCR Human Panel / changed significantly $(\mathrm{p}<0.05)$ because of the intervention. Four of these miRNAs (miR-146a, -30e, -22 and -20a) changed in all 12 samples. These miRNAs and four others (miR$19 b,-101,-505$ and $-378 a ; p<0.07)$, which also changed in all samples, were then analyzed in the sports intervention study. Furthermore, Line-1 methylation $(p=0.003)$ increased significantly.

\subsection{Intervention study $2019-2020$}

The selected miRNAs and DNA methylation sites were analyzed in the control group and intervention group before (T0) and after ( $\mathrm{T} 1$ ) 12 weeks and to assess the persistent consequence of the intervention, analysis was also done 10 months after the intervention (T2) in the follow-up group. SNP genotyping was only carried out in the intervention group.

\subsubsection{Results of the selected miRNAs and Line-1 methylation}


Intervention group: Five of the selected miRNAs showed an altered expression level after the sports intervention. Three miRNAs (miR-20a, $p=0.017,0.95$-fold; miR-22, $p=0.012,0.95$-fold and miR-505, $p=$ $0.006,0.95$-fold) were significantly downregulated and two (miR-30e, $p=0.075,0.97$-fold and miR-146a, $p$ $=0.066,0.97$-fold) showed a downregulation trend (Table 1). However, to quantify the effect of the intervention, we evaluated a "fitness sore", which depends on the strength / p-values of the individual miRNAs in the intervention and control group. Whereby a high score means better fitness and as can be seen in Figure 2, the "fitness score" increased significantly in the entire intervention group $(p=0.000)$, in men $(p=0.005)$ and women $(p=0.009)$, while it did not change in the control group $(p=0.740)$. Furthermore, the methylation of Line-1 showed no change after 12-week intervention.

Control group: In the control group, only one miRNA had a changed expression after 12 weeks. The miR20 a showed a significant upregulation ( $p=0.020,1.48$-fold), whereas in the intervention group a significant downregulation was observed. When the intervention and the control group are compared, they showed a significantly different expression of miR-20a $(p=0.042)$ before the intervention (T0) and significantly different expressions of miR-22 $(p=0.000),-30 e(p=0.015)$ and $-146 a(p=0.047)$ after 12 weeks (T1). If considering the fold changes of the two groups, they differ significantly in miR-20a ( $p=$ $0.012)$ and $\operatorname{miR}-22(p=0.046)$.

Follow-up group: In the follow-up group we observed an upregulated miR-378a 10 months after the sports intervention (T2), compared to immediately after the intervention (T1) $(p=0.050)$. When comparing before the intervention (T0) and 10 months after the intervention (T2), there are no differences in the expression levels of these miRNAs.

\subsubsection{Results of the SNP genotyping}

Table 2 shows the distribution of the selected single nucleotide polymorphism (SNPs) in the respective genes of our study participants in the intervention group $(n=61)$.

Differences between genotypes and selected miRNAs: Polymorphisms in the ACE gene seem to influence the expression of miR-101 $(p=0.019)$. Participants with a CC genotype had a significantly higher expression after the intervention compared to participants with a CG or GG phenotype, with no differences between CG and GG (Figure 3A). Furthermore, polymorphisms in the ACTN3 gene appear to have an influence on the expression of miR-20a $(p=0.027)$, miR-22 ( $=0.033)$ and miR-101 ( $p=0.004)$, see Figure 3B-D. Participants with a CC genotype had a significant higher expression of these miRNAs after 12 weeks than those with a TC genotype. Whereas the participants with a TT genotype showed a higher expression of miR-101 than TC genotypes. Furthermore, the polymorphism in the FTO gene also seems to influence the expression of miR-22 $(p=0.008)$. However, only the $G G$ genotype differed from the AA genotype. Participants with a GG genotype showed a higher expression after the intervention than those with an AA genotype (Figure 3E).

\subsubsection{Results of the body composition measurement (BIA)}


The Intervention resulted in significant changes in the anthropometric data. There was an increase in metabolic rate at rest $(p=0.000)$, phase angle $(p=0.004), \operatorname{LBM}(p=0.000), B C M(p=0.007)$ as well as ICW $(p=0.007)$ and ECW $(p=0.015)$, and a decrease in BFM $(p=0.000)$, see Table 3.

Epigenetic and genetic markers and anthropometric data: We were also able to determine significant correlations between miRNAs, Line-1 methylation and anthropometric data. Between miR-22 (linear regression $=-0.277, p=0.031$ ), miR-101 and LBM (linear regression $=-0.267, p=0.038$ ). Among miR-505 and BFM (linear regression $=0.290, p=0.024$ ), between Line-1 methylation and ECW (linear regression $=$ $-0.267, p=0.037$ ) and among the fitness score and BFM (linear regression $=-0.343, p=0.007$ ), see Figure 4. For example, the participants who gained more LBM had a lower expression of miR-22 (Figure 4A) and miR-101 (Figure 4C), and participants who lost more BFM had a lower expression of miR-505 and a higher fitness score (Figure 4B and D).

Furthermore, we could observe correlations between ACTN3 gene, LBM $(p=0.039), \operatorname{ICW}(p=0.040)$ and BMI $(p=0.072)$. The participants with a TC genotype showed a higher gain in LBM and ICW compared to participants with a TT genotype.

\subsubsection{Results of epigenetic markers, nutrition and lifestyle}

In the intervention group, we were able to determine a significant correlation between the intake of $B$ vitamins (B vitamin complex, cobalamin and folic acid) and miR-19b ( $p=0.033)$ and miR-101 $(p=$ 0.047). Participants who supplemented with $B$ vitamins had a higher expression of these miRNAs compared to participants who did not supplement. A similar correlation could be established with the intake of magnesium and miR-19b ( $p=0.052)$. Furthermore, we were able to observe an association between miR-378a and iron supplementation $(p=0.017)$. The participants who supplemented iron had a significant higher expression of miR-378a. In addition, the participants who had a higher fluid intake (2-3 liters a day) showed an upregulation of miR-378a $(p=0.003)$. Furthermore, the participants who reported consuming whole grain products daily or several times a day showed higher expression level of miR-101 $(p=0.002)$ than those who consumed less. Whereas miR-146a correlated with stress $(p=0.029)$. The participants who reported having an increased stress level had a significantly lower expression of miR$146 a$.

\section{Discussion}

The main aim of this study was to measure training effects via miRNAs from dried blood spots (DBS) and to determine how miRNAs are regulated by medium-term, according to Ashton et al. 2020 [19], exercise training (strength and endurance) in sedentary, healthy individuals. But also, what role genetic predisposition or nutrition plays and the possible interactions with the selected miRNAs, as well as the development of a biomarker algorithm that provides information about the performance, health and recovery of an athlete. 
As far as we know, we are among the first to describe a so-called miRNA-based "fitness score" biomarker in capillary blood, which can describe a person's current fitness level regardless of age, gender and genetic predisposition. And even if some of the miRNAs analyzed in this study have already been described in combination with exercise and could be used as biomarkers such as miR-20a and -146a for cardiorespiratory fitness and peak exercise capacity [20], a combination of several miRNAs promises a more precise assessment of health and training levels. Also, with the currently commonly used biomarkers, it was found that a single maker alone is to meaningless and that a combined analysis of several parameters provides much more accurate information and thus helps athletes or coaches to optimize training and prevent overtraining [16]. Furthermore, using a miRNA-based biomarker algorithm would have the further advantage that the miRNA expression patterns changes much earlier than markers from proteins and / or electrolytes [17]. For this purpose, it should also be considered which information our miRNAs provide, which sport-relevant physiological functions they may reflect or have and where they come from.

It is known that exercise training leads to an increased rate of red blood cell (RBC) breakdown due to oxidative stress. However, as a result, exercise training stimulates erythropoiesis and increases RBC mass, the number of leukocytes, hematocrit values and hemoglobin concentrations, as well as the plasma volume. And, an increased rate of RBC turnover can be beneficial as the young cells are more efficient at transporting oxygen [21-23]. Moreover, it was found that RBCs are the leading cause of miRNA expression in whole blood and that the amount of erythrocyte-derived miRNAs reflects the majority of miRNAs expressed in the whole blood. It is described that these RBC-derived miRNAs show high expression patterns and some are delivered to recipient cells via extracellular erythrocyte vesicles and are thus involved in the regulation of gene expression. However, the amount of the endogenous controls (miR-24 and -93) in our DBS samples did not change during the intervention and we could not find any indications in the literature that they are involved in the regulation of erythropoiesis in healthy humans. Moreover, there are other important cell types in whole blood, such as leukocytes and platelets, that contain functional miRNAs [24].

As already indicated, exercise also affects leukocytosis, increases the number of leukocytes and neutrophils. And it is believed that these changes in the hemodynamics of the blood cells take place in the circulatory system and may not weaken the immune system, but actually strengthen it due to the increased number of immune cells. Although exercise can resemble acute inflammation by recruiting leukocytes, exercise could be beneficial as most cells are recruited into the bloodstream rather than into inflamed tissues, as in infection [25]. It is possible that the analyzed miRNAs come from the leukocytes or neutrophils, since exercise can also alter the miRNA expression patterns in neutrophils in the circulating blood and thus possibly influence the function of the neutrophils [26]. Furthermore, it was shown that miR-20a and -22 were downregulated in neutrophils after endurance training, while miR-505 was upregulated, and miR-30e in natural killer cells and monocytes. Some of the neutrophil-derived miRNAs, including miR-20a, are also known to regulate genes involved in immune processes and apoptosis, such as Jak-STAT signaling pathways [15,26-28]. Also miR-146a plays an essential role in the inflammatory signaling in several cell types and it was concluded that changes in the circulating miR-146a could reflect 
the overall inflammatory state of prolonged aerobic exercise [29]. Moreover this miRNA also correlates with the inflammation marker high-sensitivity C-reactive protein [30]. In summary this suggests that some of our miRNAs play a role in the exercise-influenced immune system.

However, we were able to determine differences in the expression of miR-20a, -22 and -101 depending on the a-actinin-3 gene (ACTN3), often also referred to as the "speed gene". Subjects with a CC (or RR) genotype had a higher expression of miR-20a and -22 after 12-weeks than those with a TC (or XR) genotype, while participants with a TT (or XX) genotype had a higher expression of miR-101 than those with a TC or CC genotype. The protein ACTN3 is only expressed in the fast type II fibers and is related to the generation of fast and powerful muscle movements. It also appears to play a role in adaption and recovery / regeneration after exercise and in the risk of injury. In contrast to individuals with TC or CC, those with homozygous TT cannot express ACTN3, which can lead to impaired muscle performance and make them predisposed to muscle damage and ligament injuries during exercise training [7]. In animal studies, but also in some human studies, especially in women, this phenotype has been also associated with increased endurance capacity [31-33]. Our results indicate that there may be an association between the expression of miR-20a,-22 and -101, the genotype of ACTN3 and the ability to perform fast and explosive movements and / or the injury risk. Additionally, we were able to observe a correlation between miR-22, -101 and the increase of lean body mass, which further confirms a possible connection to springiness. It is also discussed that these miRNAs are involved in cardiac hypertrophy trigger by endurance exercise and we suspected that they may also have pro- / anti-hypertrophic effects in other tissues [34]. Furthermore, participants with the FTO risk allele (AA) the expression of miR-22 was downregulated. One known target of miR-22 is the histone deacetylase 4 (HDAC4) [35], supporting the theory that metabolic phenotypes probably mediated by epigenetic mechanisms due to the different FTO mutations [36]. These results highlight the importance of personalized analysis and interventions, as the epigenetic phenotype varies significantly depending in the genotype.

Anyway, it is known that exercise-induced muscle injuries and damage often occur after unusual or intense training, especially if the training involves many eccentric contractions [37,38]. In response to damage, activated satellite cells begin to multiply and undergo myogenic differentiation as part of the muscle regeneration process. The proliferation and differentiation of satellite cells is associated with changes in miRNA expression, which leads to an altered expression of target genes that are important for muscle regeneration. These muscle-specific miRNAs also include miR-378a, which has been identified as a promising marker for acute muscle damage $[39,40]$. Furthermore, miR-378a is a positive regulator of osteogenesis and is therefore involved in bone formation and resorption, making miR-378a a marker of bone health and injury / bone fracture risk [41,42]. Other miRNAs are also involved in the regulation of bone remodeling and regeneration, such as miR-146a, which, in contrast to miR-378a, suppresses osteoblastogenesis [43] and miR-30e, which inhibits osteoblast differentiation, whereby its expression level maintains the balance between osteoblast and adipocyte differentiation [44]. Bone marrow adipocytes influence bone remodeling and play a role in bone loss disorders [45]. However, exercise can suppress bone marrow adipocytes expansion [46], maybe through miR-30e. 
Besides these, in our study, we were also able to observe a correlation between the self-reported supplementation of B vitamins, which include thiamine, riboflavin, niacin, pantothenic acid, pyridoxine, biotin and folic acid or folate, and miR-19b expression. An intake of B vitamins leads to an upregulation of miR-19b. Furthermore, we could also observe that the participants who supplemented magnesium had a higher expression of miR-19b and those who consumed iron supplements had a higher expression of miR-378a. Very little evidence of such correlations can be found in the literature. But it is known that micronutrients are essential for maintaining the health of physically active people and that especially $B$ vitamins are essential for energy production, synthesis of new cells and repair of damaged cells. Whereas others are necessary for maintaining the immune function, bone health and protecting against oxidative damage, but also for building and repairing muscle tissue. Both biochemical adaptions to exercise and the metabolic pathways for energy production can increase the need for certain micronutrients during regular physical activity and athletes with a poor B vitamin status may have a decreased ability to exercise at a high intensity level [47-49]. Furthermore, it is important particularly for athletes to maintain an optimal micronutrient status and, if necessary, to recognize a deficiency at an early stage before physical signs appear. Furthermore, the miR-378a expression correlated with the amount of average fluid intake. During exercise, especially at high temperatures, sweating and inadequate hydration can lead to a water deficit [16]. And there are indications that moderate hypohydration, even in short phases, can lead to disturbance of cognitive functions, a reduction in the ability to concentrate as well as headaches or fatigue and thus impair the performance of an athlete [50]. In summary, the correlations between micronutrients, fluid intake and miRNAs observed in our study indicate that some miRNAs are suitable for reflecting the current micronutrient and hydration status of an athlete, as predictive Biomarker.

\section{Conclusion}

In conclusion, our "fitness score" not only reflects the effect of the intervention, moreover the individual miRNAs provide information to assess the health, performance and recovery of an athlete / person. Based on our findings, correlations between miRNAs, SNPs, body composition and diet, and the literature, we have divided the miRNAs, according to the work by Lee et al., 2017 [16], into the following subgroups (Figure 5): nutrition, inflammation, cardiovascular fitness, injury risk, regeneration, muscle- and hydration status as well as stress level. As a result, our biomarker enables easier training tracking, as samples can be taken anytime and anywhere (DBS), and athletes and coaches can make possible adjustments at an early stage in order to optimize performance or prevent deficits.

\section{Abbreviations}

ACE: Angiotensin-converting enzyme

ACTN3: Alpha-actin-3

AUC: Area under the curve 
BCM: Body cell mass

BFM: Body fat mass

BIA: Bioelectrical impedance analysis

BMl: Body mass index

DBS: Dried blood spot

ECW: Extracellular water

FFQ: Food frequency questionnaire

FTO: Fat mass and obesity associated gene

HRM: High resolution melting

ICW: Intracellular water

LBM: Lean body mass

miRNA: microRNA

MREs: miRNA response elements

qPCR: Real-time polymerase chain reaction

RBC: Red blood cell

SNP: Single nucleotide polymorphism, Single nucleotide polymorphism

\section{Declarations}

\section{Acknowledgements}

Our special thanks go to the ISC Gym Fitnesscenter Vienna for providing their training rooms and equipment as well as their expertise. A big thank also goes to the participants in this study.

\section{Funding}

The experiment was supported by two FEMtech grants (No 880965; No 871016), from the Austrian Research Funding Service FFG, and financed by the Cancer Aid Foundation, Swiss, in partnership with HealthBioCare $\mathrm{GmbH}$.

\section{Author information}




\section{Affiliations}

Department of Nutritional Sciences, University of Vienna, Vienna, Austria

U. D. B. Krammer, S. Tschida, J. Berner, S. Lilja, P. Rust, A. G. Haslberger

HealthBioCare GmbH, Vienna, Austria

O. J. Switzeny, B. Hippe

\section{Contributions}

All authors conceived and designed the study, U. D. B. Krammer, S. Tschida and J. Berner conducted the experiments and analyzed the data, U. D. B. Krammer, O. J. Switzeny, B. Hippe and A. G. Haslberger interpreted the results of the experiments, U. D. B. Krammer designed the manuscript. All authors have made critical contributions, have read and approved the final manuscript.

\section{Corresponding author}

Correspondence to Alexander G. Haslberger

\section{Ethics declarations}

\section{Ethics approval and consent to participate}

No ethics approval was required for this non-invasive intervention study. Written consent was obtained from the participants for the publication of this study.

\section{Consent for publication}

All authors have consented to the contents of this paper and to the submission guidelines of the Journal of the International Society of Sports Nutrition.

\section{Competing interests}

The authors declare no conflict of interest.

\section{References}

1. Mikkelsen K, Stojanovska L, Polenakovic M, et al. Exercise and mentabnl health. Maturitas 2017; 106:48-56

2. Radak Z, Torma F, Berkes I, et al. Exercise effects on physiological function during aging. Free Radic. Biol. Med. 2019; 132:33-41

3. Distefano G, Goodpaster BH. Effects of exercise and aging on skeletal muscle. Cold Spring Harb. Perspect. Med. 2018; 8: 
4. Ling C, Rönn T. Epigenetic adaptation to regular exercise in humans. Drug Discov. Today 2014; 19:1015-1018

5. Kelly SA, Pomp D. Genetic determinants of voluntary exercise. Trends Genet. 2013; 29:348-357

6. Herring MP, Sailors MH, Bray MS. Genetic factors in exercise adoption, adherence and obesity. Obes. Rev. 2014; 15:29-39

7. Del Coso J, Hiam D, Houweling P, et al. More than a 'speed gene': ACTN3 R577X genotype, trainability, muscle damage, and the risk for injuries. Eur. J. Appl. Physiol. 2019; 119:49-60

8. Heffernan SM, Stebbings GK, Kilduff LP, et al. Fat mass and obesity associated (FTO) gene influences skeletal muscle phenotypes in non-resistance trained males and elite rugby playing position. BMC Genet. 2017; 18:1-9

9. Almén MS, Jacobsson JA, Moschonis G, et al. Genome wide analysis reveals association of a FTO gene variant with epigenetic changes. Genomics 2012; 99:132-137

10. Grazioli E, Dimauro I, Mercatelli N, et al. Physical activity in the prevention of human diseases: Role of epigenetic modifications. BMC Genomics 2017; 18:

11. Voisin S, Eynon N, Yan X, et al. Exercise training and DNA methylation in humans. Acta Physiol. 2015; 213:39-59

12. McGee SL, Hargreaves M. Epigenetics and Exercise. Trends Endocrinol. Metab. 2019; 30:636-645

13. Kern F, Ludwig N, Backes C, et al. Systematic Assessment of Blood-Borne MicroRNAs Highlights Molecular Profiles of Endurance Sport and Carbohydrate Uptake. Cells 2019; 8:

14. Wang H, Liang Y, Li Y. Non-coding RNAs in exercise. Non-coding RNA Investig. 2017; 1:10-10

15. Silva GJJ, Bye A, el Azzouzi $\mathrm{H}$, et al. MicroRNAs as Important Regulators of Exercise Adaptation. Prog. Cardiovasc. Dis. 2017; 60:130-151

16. Lee EC, Fragala MS, Kavouras SA, et al. Biomarkers in sports and exercise: tracking health, performance, and recovery in athletes. J. strength Cond. Res. 2017; 31:2920

17. Moldovan L, Batte KE, Trgovcich J, et al. Methodological challenges in utilizing miRNAs as circulating biomarkers. J. Cell. Mol. Med. 2014; 18:371-390

18. Marques-Rocha JL, Milagro FI, Mansego ML, et al. LINE-1 methylation is positively associated with healthier lifestyle but inversely related to body fat mass in healthy young individuals. Epigenetics $2016 ; 11: 49-60$

19. Ashton RE, Tew GA, Aning JJ, et al. Effects of short-term, medium-term and long-term resistance exercise training on cardiometabolic health outcomes in adults: Systematic review with metaanalysis. Br. J. Sports Med. 2020; 54:341-348

20. Baggish AL, Hale A, Weiner RB, et al. Dynamic regulation of circulating microRNA during acute exhaustive exercise and sustained aerobic exercise training. J. Physiol. 2011; 589:3983-3994

21. Smith JA. Exercise, Training and Red Blood Cell Turnover. Sport. Med. 1995; 19:9-31

22. Hu M, Lin W. Effects of exercise training on red blood cell production: Implications for anemia. Acta Haematol. 2012; 127:156-164 
23. Belviranli M, Okudan N, Kabak B. The Effects of Acute High-Intensity Interval Training on Hematological Parameters in Sedentary Subjects. Med. Sci. 2017; 5:15

24. Sun L, Yu Y, Niu B, et al. Red Blood Cells as Potential Repositories of MicroRNAs in the Circulatory System. Front. Genet. 2020; 11:1-8

25. Sand KL. Effects of exercise on leukocytosis and blood hemostasis in 800 healthy young females and males. World J. Exp. Med. 2013; 3:11

26. Radom-Aizik S, Zaldivar F, Oliver S, et al. Evidence for microRNA involvement in exercise-associated neutrophil gene expression changes. J. Appl. Physiol. 2010; 109:252-261

27. Radom-Aizik S, Zaldivar F, Haddad F, et al. Impact of brief exercise on peripheral blood NK cell gene and microRNA expression in young adults. J. Appl. Physiol. 2013; 114:628-636

28. Radom-Aizik S, Zaldivar FP, Haddad F, et al. Impact of brief exercise on circulating monocyte gene and microRNA expression: Implications for atherosclerotic vascular disease. Brain. Behav. Immun. 2014; 39:121-129

29. Baggish AL, Park J, Min PK, et al. Rapid upregulation and clearance of distinct circulating microRNAs after prolonged aerobic exercise. J. Appl. Physiol. 2014; 116:522-531

30. Li Y, Yao M, Zhou Q, et al. Dynamic regulation of circulating microRNAs during acute exercise and long-term exercise training in basketball athletes. Front. Physiol. 2018; 9:1-11

31. Roth SM, Walsh S, Liu D, et al. The ACTN3 R577X nonsense allele is under-represented in elite-level strength athletes. 2008; 16:391-394

32. Yang N, MacArthur DG, Gulbin JP, et al. ACTN3 genotype is associated with human elite athletic performance. Am. J. Hum. Genet. 2003; 73:627-631

33. Yang R, Shen X, Wang Y, et al. ACTN3 R577X gene variant is associated with muscle-related phenotypes in elite Chinese sprint/power athletes. 2017; 31:1107-1115

34. Soplinska A, Zareba L, Wicik Z, et al. MicroRNAs as biomarkers of systemic changes in response to endurance exercise $\Leftrightarrow$ a comprehensive review. Diagnostics 2020; 10:1-17

35. Lu W, You R, Yuan X, et al. The microRNA miR-22 inhibits the histone deacetylase HDAC4 to promote TH17 cell-dependent emphysema. Nat. Immunol. 2015; 16:1185-1194

36. Franzago M, Fraticelli F, Marchioni M, et al. Fat mass and obesity-associated (FTO) gene epigenetic modifications in gestational diabetes: new insights and possible pathophysiological connections. Acta Diabetol. 2021; 58:997-1007

37. Clarkson PM, Hubal MJ. Exercise-induced muscle damage in humans. Int. J. Sports Med. 2002; 15:132-135

38. Siracusa J, Koulmann N, Sourdrille A, et al. Phenotype-specific response of circulating miRNAs provides new biomarkers of slow or fast muscle damage. Front. Physiol. 2018; 9:1-9

39. Kirby TJ, McCarthy JJ. MicroRNAs in skeletal muscle biology and exercise adaptation. Free Radic. Biol. Med. 2013; 64:95-105 
40. Siracusa J, Koulmann N, Bourdon S, et al. Circulating miRNAs as Biomarkers of Acute Muscle Damage in Rats. Am. J. Pathol. 2016; 186:1313-1327

41. Kang M, Huang CC, Lu Y, et al. Bone regeneration is mediated by macrophage extracellular vesicles. Bone 2020; 141:

42. Amir LR, Everts V, Bronckers ALJJ. Bone regeneration during distraction osteogenesis. Odontology 2009; 97:63-75

43. Chang CC, Venø MT, Chen L, et al. Global MicroRNA Profiling in Human Bone Marrow SkeletalStromal or Mesenchymal-Stem Cells Identified Candidates for Bone Regeneration. Mol. Ther. 2018; 26:593-605

44. Suttamanatwong S. MicroRNAs in bone development and their diagnostic and therapeutic potentials in osteoporosis. Connect. Tissue Res. 2017; 58:90-102

45. Muruganandan S, Govindarajan R, Sinal CJ. Bone Marrow Adipose Tissue and Skeletal Health. Curr. Osteoporos. Rep. 2018; 16:434-442

46. Styner M, Pagnotti GM, McGrath C, et al. Exercise Decreases Marrow Adipose Tissue Through ßOxidation in Obese Running Mice. J. Bone Miner. Res. 2017; 32:1692-1702

47. Woolf K, Manore MM. B-vitamins and exercise: Does exercise alter requirements? Int. J. Sport Nutr. Exerc. Metab. 2006; 16:453-484

48. Laires MJ, Monteiro C. Exercise, magnesium and immune function. Magnes. Res. 2008; 21:92-96

49. Beard J, Tobin B. Iron status and exercise. Am. J. Clin. Nutr. 2000; 72:594-597

50. Maughan RJ. Impact of mild dehydration on wellness and on exercise performance. Eur. J. Clin. Nutr. 2003; 57:S19-S23

\section{Tables}

Table 1. Results of the analyzed markers of the participants of the sports intervention group. Results were given in mean \pm SD. 


\begin{tabular}{|c|c|c|c|c|c|c|}
\hline & \multicolumn{2}{|l|}{ Total $(n=61)$} & \multicolumn{2}{|l|}{ Male $(n=20)$} & \multicolumn{2}{|c|}{ Female $(n=41)$} \\
\hline & $\begin{array}{l}\text { Fold change } \pm \\
\text { SD }\end{array}$ & $\begin{array}{l}\text { p- } \\
\text { Value }\end{array}$ & $\begin{array}{l}\text { Fold change } \pm \\
\text { SD }\end{array}$ & $\begin{array}{l}\text { p- } \\
\text { Value }\end{array}$ & $\begin{array}{l}\text { Fold change } \pm \\
\text { SD }\end{array}$ & $\begin{array}{l}\mathrm{p}- \\
\text { Value }\end{array}$ \\
\hline miR-19b-3p & $1.212 \pm 0.764$ & 0.463 & $1.119 \pm 0.619$ & 0.641 & $1.221 \pm 0,832$ & 0.576 \\
\hline miR-20a-5p & $0.948 \pm 0.291$ & $0.017 *$ & $0.917 \pm 0.265$ & 0.083 & $0.963 \pm 0.305$ & 0.095 \\
\hline miR-22-5p & $0.945 \pm 0.315$ & $0.012^{*}$ & $0.891 \pm 0.311$ & $0.034^{*}$ & $0.971 \pm 0.318$ & 0.123 \\
\hline miR-30e-3p & $0.973 \pm 0.290$ & 0.075 & $1.010 \pm 0.330$ & 0.568 & $0.954 \pm 0.271$ & 0.072 \\
\hline miR-101-3p & $1.424 \pm 1.241$ & 0.256 & $1.744 \pm 1.537$ & 0.280 & $1.268 \pm 1.054$ & 0.621 \\
\hline miR-146a-5p & $0.967 \pm 0.297$ & 0.066 & $0.905 \pm 0.290$ & 0.114 & $0.998 \pm 0.298$ & 0.345 \\
\hline miR-378a-3p & $0.985 \pm 0.248$ & 0.172 & $0.974 \pm 0.213$ & 0.315 & $0.991 \pm 0.266$ & 0.313 \\
\hline miR-505-3p & $0.948 \pm 0.177$ & $0.006 *$ & $0.923 \pm 0.127$ & $0.011^{*}$ & $0.961 \pm 0.197$ & 0.075 \\
\hline $\begin{array}{l}\text { Line } \\
1 \text { methylation }\end{array}$ & $-0.306 \pm 4.166$ & 0.568 & $-0.448 \pm 3.818$ & 0.606 & $-0.238 \pm 4.369$ & 0.729 \\
\hline
\end{tabular}

Abbreviations: SD = standard deviation, * shows significant p-values (paired t-test).

Table 2. Distribution of the SNPs in the FTO (rs1121980), ACE (rs4341) and ACTN3 (rs1815739) gene. Genotyping values are given in total numbers and as a percentage. 


\begin{tabular}{lllll} 
Gene & SNP ID & & & \\
\hline FTO & rs1121980 & Homozygotes (GG) & Homozygotes (AA) & Heterozygotes (AG) \\
\hline & Total, \% (n) & $29.5 \%(18)$ & $24.6 \%(15)$ & $45.9 \%(28)$ \\
\hline & Male, \% (n) & $20.0 \%(4)$ & $30.0 \%(6)$ & $50.0 \%(10)$ \\
\hline \multirow{2}{*}{ ACE } & Female, \% (n) & $34.1 \%(14)$ & $22.0 \%(9)$ & $43.9 \%(18)$ \\
\hline & rs4341 & Homozygotes (CC) & Homozygotes (GG) & Heterozygotes (CG) \\
\hline & Total, \% (n) & $19.7 \%(12)$ & $31.1 \%(19)$ & $49.2 \%(30)$ \\
\hline \multirow{2}{*}{ ACTN3 } & Male, \% (n) & $20.0 \%(4)$ & $30.0 \%(6)$ & $50.0 \%(10)$ \\
\hline & rs1815739 & Homozygotes (CC) & Homozygotes (TT) & Heterozygotes (TC) \\
\hline & Total, \% (n) & $32.8 \%(20)$ & $18.0 \%(11)$ & $49.2 \%(30)$ \\
\hline & Male, \% (n) & $20.0 \%(4)$ & $30.0 \%(6)$ & $50.0 \%(10)$ \\
\hline & Female, \% (n) & $39.0 \%(16)$ & $12.2 \%(5)$ & $48.8 \%(20)$
\end{tabular}

Abbreviations: FTO = Fat mass and obesity-associated protein, ACE = Angiotensin-converting enzyme, ACTN3 = alpha-Actinin 3

Table 3. Changes in body composition during the 12-week sport intervention. Anthropometric measurements were given in mean \pm SD. 


\begin{tabular}{|c|c|c|c|c|c|c|}
\hline & \multicolumn{2}{|l|}{ Total $(n=61)$} & \multicolumn{2}{|l|}{ Male $(n=20)$} & \multicolumn{2}{|c|}{ Female $(n=41)$} \\
\hline & $\begin{array}{l}\text { Fold change } \\
\pm \text { SD }\end{array}$ & Value & $\begin{array}{l}\text { Fold change } \\
\pm \text { SD }\end{array}$ & Value & $\begin{array}{l}\text { Fold change } \\
\pm \text { SD }\end{array}$ & Value \\
\hline $\begin{array}{l}\text { Metabolic rate at rest } \\
\text { [kcal] }\end{array}$ & $23.10 \pm 41.74$ & $0.000^{*}$ & $20.45 \pm 25.67$ & $0.002^{*}$ & $\begin{array}{l}24.39 \pm \\
47.91\end{array}$ & $0.002^{*}$ \\
\hline Phase angel $\left[^{\circ}\right]$ & $0.16 \pm 0.42$ & $0.004^{*}$ & $0.07 \pm 0.25$ & 0.232 & $0.21 \pm 0.48$ & $0.009^{*}$ \\
\hline LBM [kg] & $0.68 \pm 1.39$ & $0.000^{*}$ & $0.84 \pm 1.32$ & $0.010^{*}$ & $0.60 \pm 1.43$ & $0.010^{\star}$ \\
\hline BFM [kg] & $-1.14 \pm 1.87$ & $0.000^{*}$ & $-1.33 \pm 2.19$ & $0.014^{*}$ & $-1.04 \pm 1.72$ & $0.000^{*}$ \\
\hline BCM [kg] & $0.77 \pm 2.16$ & $0.007^{*}$ & $0.64 \pm 3.17$ & 0.378 & $0.83 \pm 1.48$ & $0.001^{*}$ \\
\hline ICW [I] & $0.31 \pm 0.46$ & $0.000^{*}$ & $0.33 \pm 0.43$ & $0.003^{*}$ & $0.30 \pm 0.48$ & $0.000^{*}$ \\
\hline ECW [l] & $0.20 \pm 0.63$ & $0.015^{*}$ & $0.29 \pm 0.64$ & 0.056 & $0.16 \pm 0.63$ & 0.112 \\
\hline
\end{tabular}

Abbreviations: $\mathrm{LBM}=$ lean body mass, $\mathrm{BFM}=$ body fat mass, $\mathrm{BCM}=$ body cell mass, $\mathrm{ICW}=$ intracellular water, $\mathrm{ECW}=$ extracellular water. Fold Change $=$ mean difference between the 2 timepoints, $\mathrm{SD}=$ standard deviation, * shows significant p-values (paired t-test).

\section{Figures}




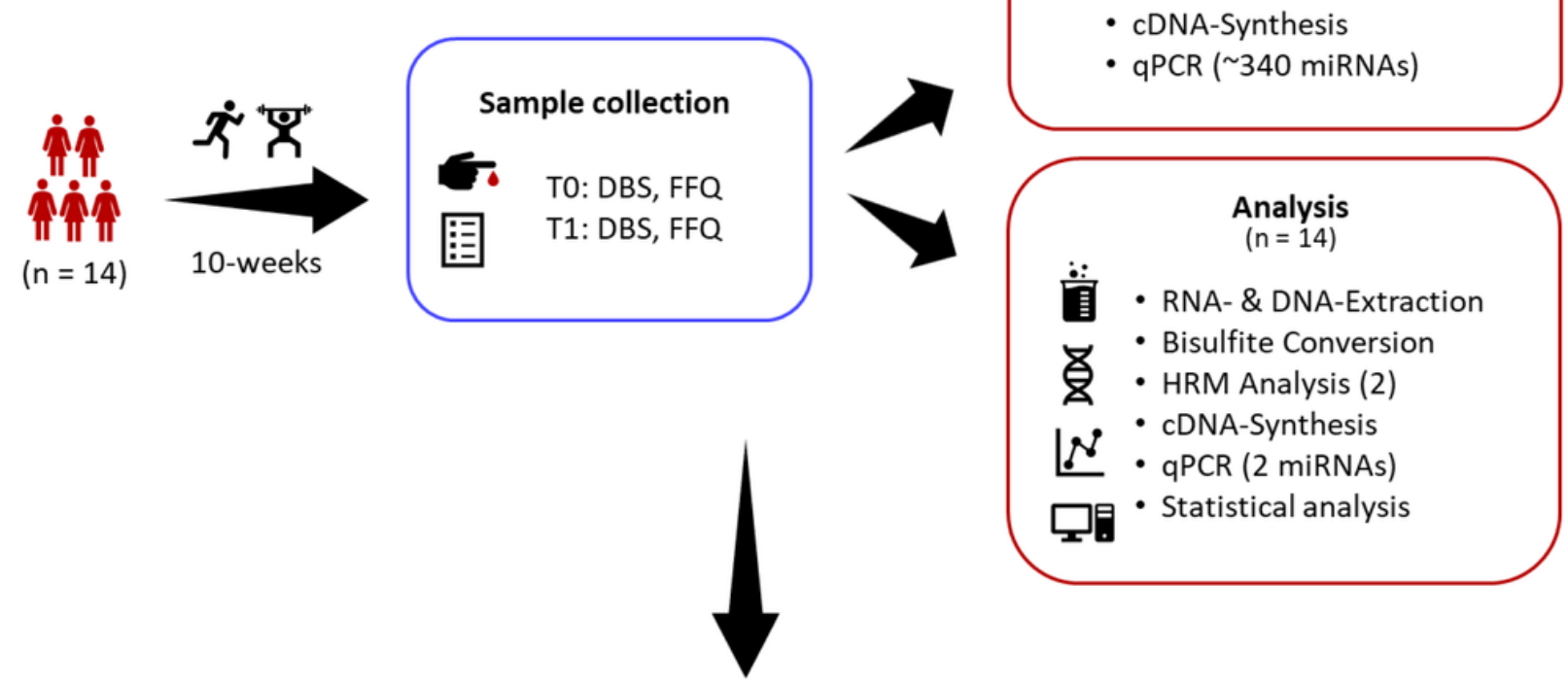

B) Intervention study 2019 - 2020

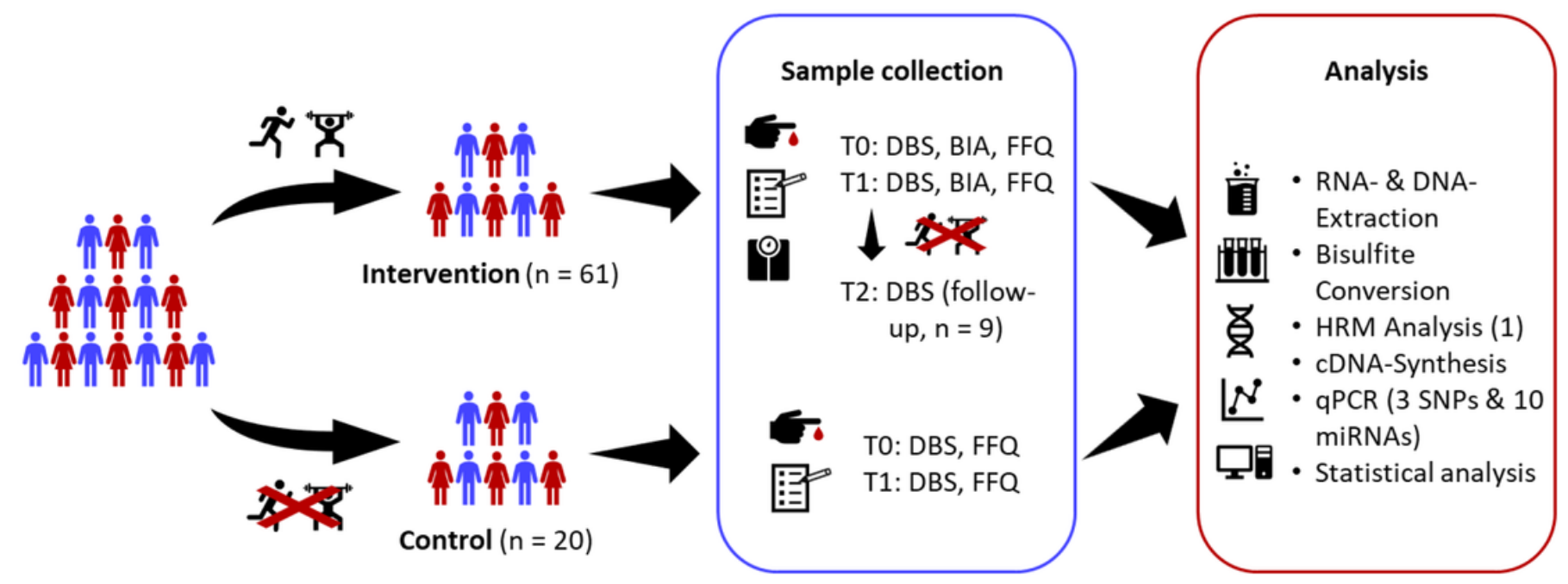

Figure 1

Study design of the pilot study 2018 (A) and the intervention study $2019-2020$ (B). T0 = begin, T1 = after 10- / 12-weeks, T2 = after 10-month, DBS = dried blood spot, BIA = bioelectrical impedance analysis, FFQ = food frequency questionnaire, HRM = high-resolution melting, SNP = single nucleotide polymorphism. 


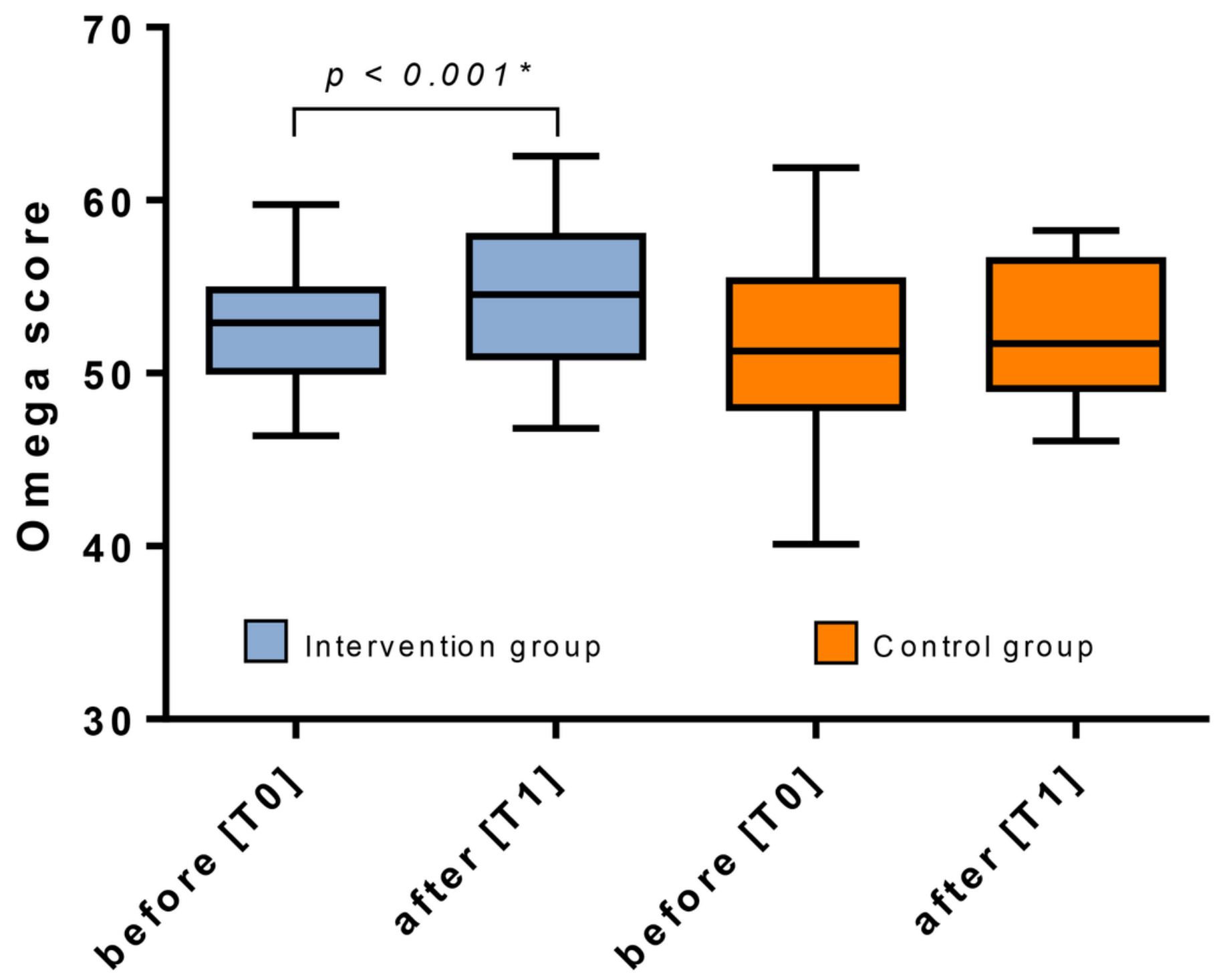

Figure 2

Fitness / $\Omega$ score of the intervention group $(n=61)$ and the control group $(n=20)$ before [T0] and after [T1]. * shows significant $p$-values (paired t-test). 
A)

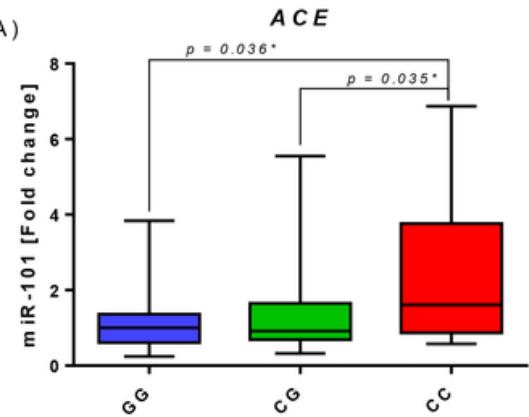

D)

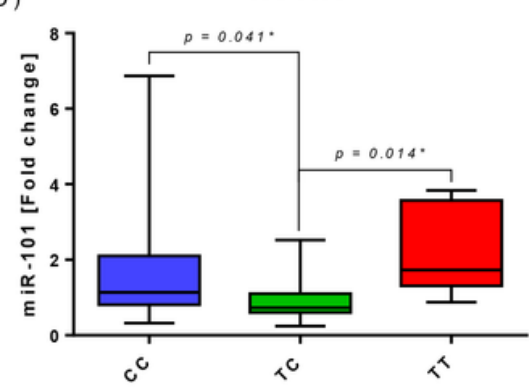

B )

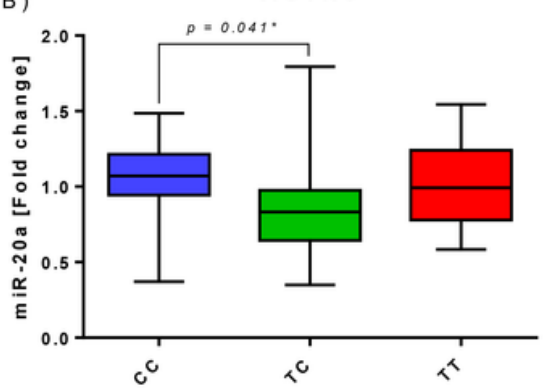

E)

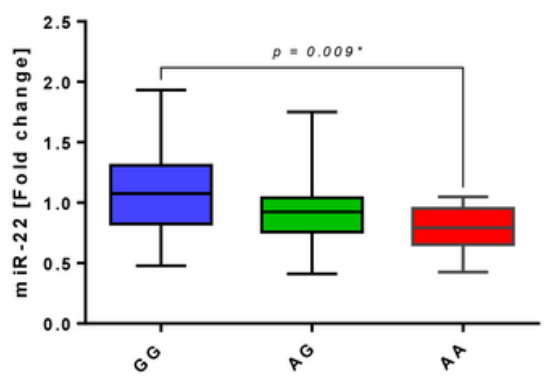

C)

ACTN 3

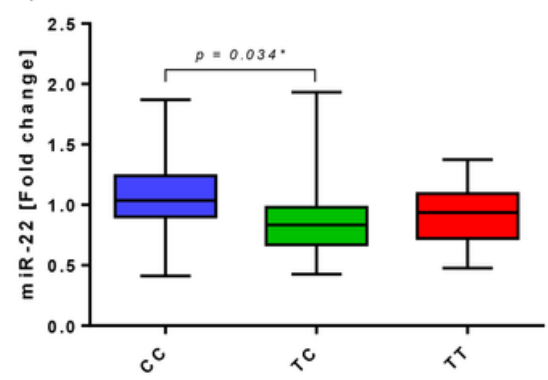

Figure 3

Fold changes of the selected miRNAs by genotype. A) Fold change of miR-101 by ACE genotype (GG strength, $C$ endurance). B) Fold change of miR-20a by ACTN3 genotype (CC strength, TT endurance). C) Fold change of miR-22 by ACTN3 genotype (CC strength, TT endurance). D) Fold change of miR-101 by ACTN3 genotype (CC strength, TT endurance). E) Fold change of miR-22 by FTO genotype (AA risk variant). The $p$-values given here are the results of the post hoc tests (Scheffé). * shows significant pvalues

A)

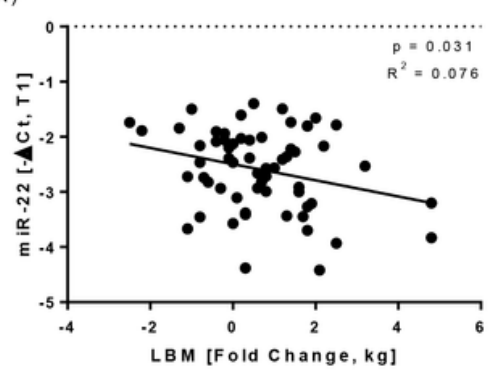

D)

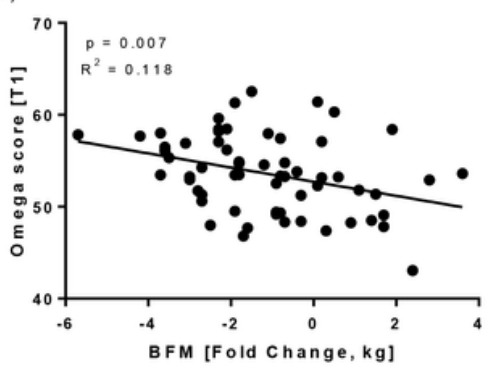

B)

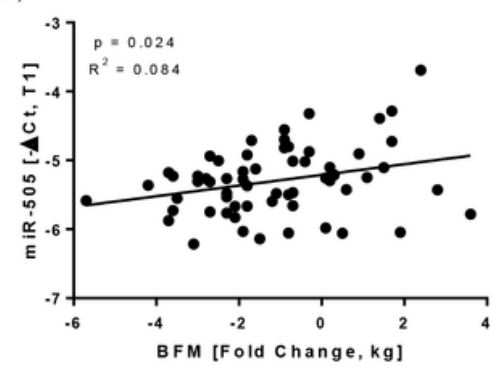

E)

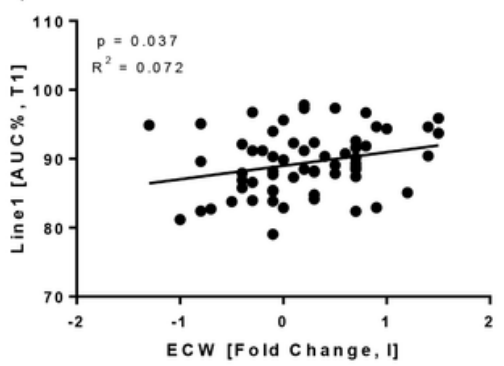

C)

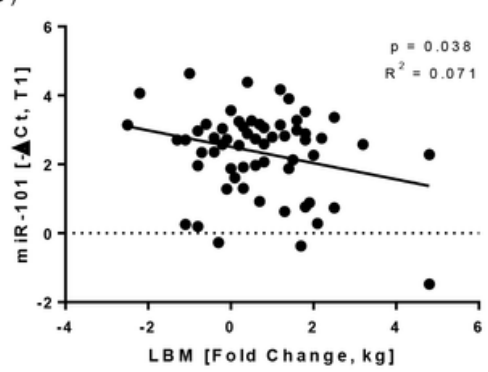

Figure 4 
Significant correlations between the change of the anthropometric data and the expression of selected miRNAs, the fitness / $\Omega$ score and Line-1 methylation after the Intervention [T1]. A) Correlation between the change of LBM and the expression of miR-22 at T1. B) Correlation between the change of BFM and the expression of miR-505 at T1. C) Correlation between the change of LBM and the expression of miR101 at T1. D) Correlation between the change of BFM and the fitness / $\Omega$ score at T1. E) Correlation between the change of ECW and the Line-1 methylation at T1. Statistical significance was defined as a pvalue below 0.05 .

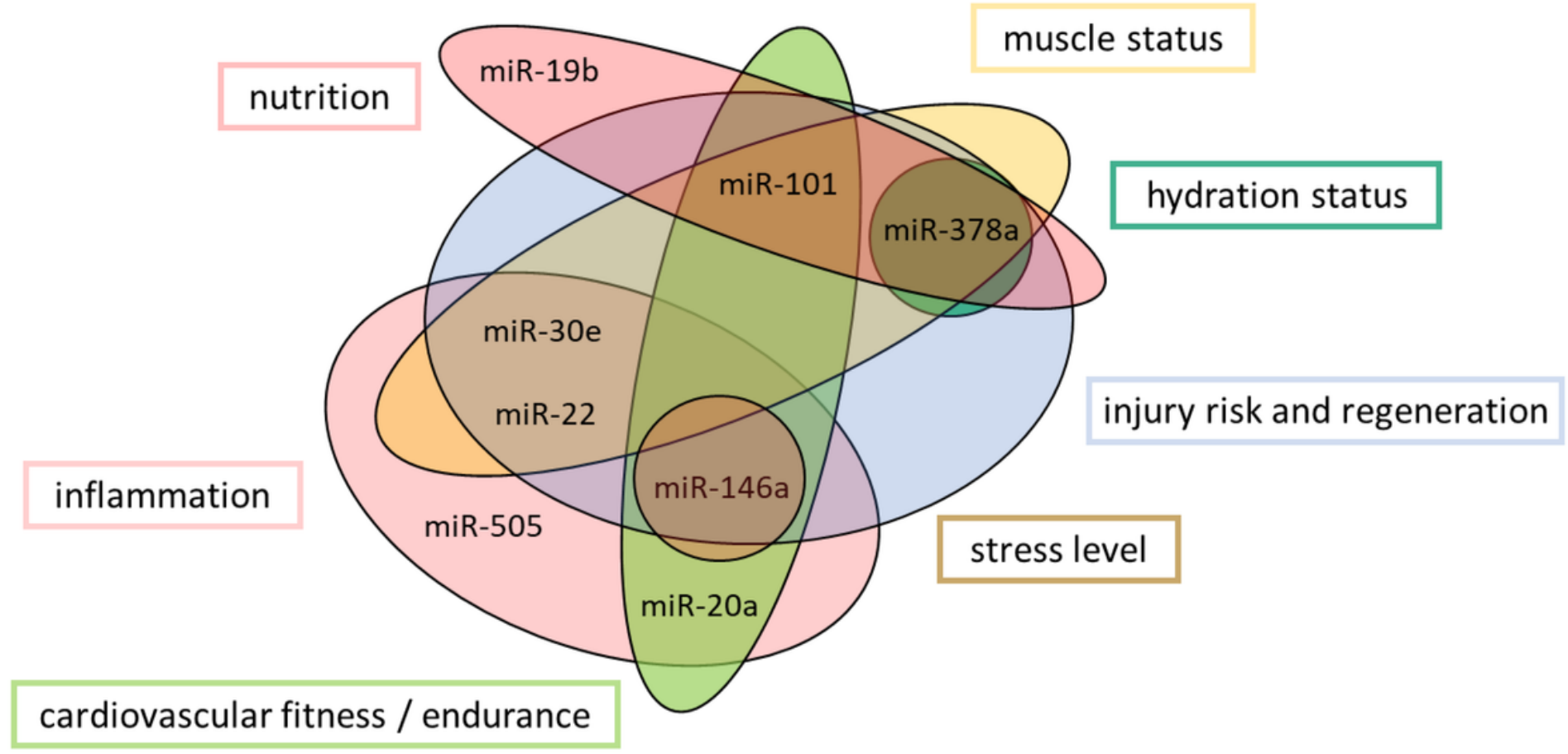

\section{Figure 5}

The properties of the individual miRNAs and their importance and classification as sports-relevant biomarkers.

\section{Supplementary Files}

This is a list of supplementary files associated with this preprint. Click to download.

- Supplements.docx 